\title{
The discovery of X-ray binaries in the Sculptor Dwarf Spheroidal Galaxy
}

\author{
Thomas J. Maccarone ${ }^{1}$, Arunav Kundu ${ }^{2}$, Stephen E. Zepf ${ }^{2}$, \\ Anthony L. Piro ${ }^{3}$ and Lars Bildsten ${ }^{3,4}$ \\ ${ }^{1}$ School of Physics and Astronomy, University of Southampton, Southampton, SO17 1BJ, UK \\ email: tjm@phys.soton.ac.uk \\ ${ }^{2}$ Department of Physiscs and Astronomy, Michigan State University, East Lansing, MI, USA \\ ${ }^{3}$ Department of Physics, University of California at Santa Barbara, Santa Barbara, CA, USA \\ ${ }^{4}$ Kavli Institute for Theoretical Physics, University of California at Santa Barbara, Santa \\ Barbara, CA, USA
}

\begin{abstract}
We report the results of a deep Chandra survey of the Sculptor dwarf spheroidal galaxy. We find five X-ray sources with $L_{X}$ of at least $6 \times 10^{33} \mathrm{ergs} / \mathrm{sec}$ with optical counterparts establishing them as members of Sculptor. These X-ray luminosities indicate that these sources are X-ray binaries, as no other known class of Galactic point sources can reach 0.5-8 $\mathrm{keV}$ luminosities this high. Finding these systems proves definitively that such objects can exist in an old stellar population without stellar collisions. Three of these objects have highly evolved optical counterparts (giants or horizontal branch stars), as do three other sources whose X-ray luminosities are in the range which includes both quiescent low mass X-ray binaries and the brightest magnetic cataclysmic variables. We predict that large area surveys of the Milky Way should also turn up large numbers of quiescent X-ray binaries.
\end{abstract}

Keywords. X-rays:binaries - X-rays:galaxies - stars:binaries:close - galaxies:individual:Sculptor dwarf spheroidal - stellar dynamics - stars:Population II.

\section{Introduction}

It is quite difficult for field star populations to produce low mass X-ray binaries (LMXBs), especially those with neutron star primaries. Supernova explosions which eject more than half the mass of a system normally leave the systems unbound. Therefore the only ways to produce LMXBs through binary stellar evolution are through common envelope evolution which ejects much of the mass of the black hole's or neutron star's progenitor before the supernova occurs (Paczynski 1976; Kalogera \& Webbink 1998), or through a finely-tuned asymmetric velocity kick which occurs at the birth of the neutron star or black hole (Brandt \& Podsiadlowski 1995; Kalogera 1998). Intermediate mass X-ray binaries (i.e. X-ray binaries where the donor star is 1-8 $M_{\odot}$ ) can lose large amounts of mass and then evolve into LMXBs, which may help to solve the problem of formation rates of such LMXBs, since these systems will be more likely to survive the supernova explosions (see e.g. Podsiadlowski, Rappaport \& Pfahl 2002). Because of the uncertainties in how to keep LMXBs bound, and other uncertainties regarding, e.g., the correlation between the initial masses of the stars in a binary system, theoretical rates of X-ray binary formation are highly uncertain.

The difficulties in producing LMXBs are even larger for old stellar populations. The mass accretion rate required to power a persistent bright LMXB is about $10^{-8} M_{\odot} \mathrm{yr}^{-1}$, meaning that the lifetime of such a system can be only about 100 Myrs from when accretion starts. It is generally believed that most LMXBs will begin their accretion phases 
only a few Gyrs after the supernova which creates the compact object (White \& Ghosh 1998). Several possibilities still exist for producing X-ray binaries in old populations such as elliptical galaxies - these LMXBs we see in old stellar populations may be very low duty cycle transients (meaning that they will take a long time to accrete their entire mass donors) which begin accretion only after their donor stars evolve off the main sequence (Piro \& Bildsten 2002), they may be ultracompact X-ray binaries (Bildsten \& Deloye 2004), or they may be normal X-ray binaries which were produced through dynamical encounters in globular clusters, and then released into the field (e.g. White, Sarazin \& Kulkarni 2002; see also Grindlay 1988).

If the bulk of the elliptical galaxy field sources are low duty cycle transients, then there must exist an underlying population of quiescent X-ray binaries which is much larger than the fraction which is flaring at any given time. Therefore, we have identified a large sample of old stars which is nearby, and does not contain any globular clusters, the Sculptor dwarf spheroidal galaxy. By finding five X-ray binaries in this galaxy, four of which have highly evolved donor stars, we have verified that the mechanism of Piro \& Bildsten (2002) is, at the very least, a substantial contributor to the X-ray binary populations seen in old field star populations.

\section{Data}

We have obtained 21 exposures of 6-kiloseconds each with the Chandra X-ray observatory over the time period from 26 April 2004 to 10 January 2005. The monitoring, rather than a single deep observation, was performed in order to search for bright transient sources, but none were found. We then stacked the data to make a single image of the data on ACIS-S3, and ran WAVDETECT, finding 74 sources in the $0.5-8.0 \mathrm{keV}$ band. Based on previous deep field measurements (e.g. Hornschemeier et al. 2001), we estimate that about 50 of these sources should be background AGN and that it is unlikely that more than one is a foreground star. In order to determine whether there are supersoft X-ray sources, we have also extracted an image from $0.1-0.4 \mathrm{keV}$, but this image contained no bright sources (i.e. with more than 10 photons) that were not found in the 0.5-8.0 keV image.

We have compared the positions of the 9 sources with at least 100 detected counts (giving accurate positions, and making them bright enough to rule out the possibility of a white dwarf accretor) with the positions of bright optical stars $(V<20.5)$ in the Sculptor galaxy from Schweitzer et al. (1995). The stars in the Schweitzer et al. (1995) catalog are all red giants, asymptotic giant branch stars, or horizontal branch stars. We have also checked whether the single brightest source has an optical counterpart in the deep optical photometry of Hurley-Keller, Mateo \& Grebel (1999).

Allowing for an 0.6 " boresight correction we find four matches within 0.4 " of an optical star in Schweitzer et al. (1995). The chance superposition probability is 0.04 for these objects, and all have proper motion confirmations that they are members of Sculptor, rather than foreground or background sources. The fifth match is the brightest X-ray source in our sample, which is found at $\mathrm{RA}=1 \mathrm{~h} 00 \mathrm{~m} 13.9 \mathrm{~s}, \mathrm{Dec}=-33 \mathrm{~d} 44 \mathrm{~m} 42.5 \mathrm{~s}$. While there is no strong evidence for it to be variable in our monitoring campaign, it was not detected in the ROSAT bright source all-sky survey, so it must either be variable at the level of a factor of a few, or it must have a very hard spectrum at soft X-rays. Its optical counterpart is $R=23.68, B-R=0.95$ (D. Hurley-Keller private communication - see Hurley-Keller et al. 1999 for a description of how the photometry was done), giving it a luminosity and color roughly consistent with a solar-type star in the low metallicity 
environment of the Sculptor galaxy, and it appears to be near the turnoff of the main sequence in the Sculptor galaxy.

\section{Discussion}

\subsection{The nature of the matches}

It has been suggested that the field populations of elliptical galaxies are low duty cycle transients, with red giant donors (Piro \& Bildsten 2002). Obviously the finding that several of these systems have red giant donors provides support for this hypothesis. The number of bright (i.e. $L_{X}>10^{37} \mathrm{ergs} / \mathrm{sec}$ ) LMXBs per unit stellar mass seems to be roughly constant across a sample of giant elliptical galaxies (Gilfanov 2004), with a typical value of about one per $10^{8}-10^{9} M_{\odot}$ of stars. We would then expect about $\sim .02$ such systems in Sculptor, given its mass of about $2 \times 10^{6} M_{\odot}$ (Mateo 1998). Given that the duty cycles of these transients are likely to be less than about $1 / 200$ in the scenario of Piro \& Bildsten (2002), one would expect that the number of quiescent transient X-ray binaries in the Sculptor galaxy would be at least a few, and possibly much higher if the duty cycles were even smaller than $1 / 200$. Our discovery of one clear case of an X-ray binary with a red giant counterpart and a few more candidates is thus in reasonable agreement with the picture suggested by Piro \& Bildsten (2002).

\subsection{Are there other quiescent $X$-ray binaries in Sculptor?}

It is likely that there are actually many more quiescent X-ray binaries in the Sculptor galaxy than the five strong matches we have presented here and the three additional tentative matches. Our detection limits in this data set are at a luminosity of a little bit above $10^{32} \mathrm{ergs} / \mathrm{sec}$, while quiescent LMXBs have been seen to be as faint as $2 \times$ $10^{30} \mathrm{ergs} / \mathrm{sec}$, with most of the faintest sources having black hole accretors (Garcia et al. 2001). Therefore, some quiescent X-ray binaries could be below our sensitivity limits, although most neutron star accretors are brighter than these limits in quiescence, and there do seem to be higher quiescent luminosities for long period transients, even for black hole accretors. There may also be other quiescent X-ray binaries which are detected in our observations, but which have faint optical counterparts. These could be, for example, ultracompact X-ray binaries, which have also been suggested to be systems which should be present in old stellar populations (Bildsten \& Deloye 2004), or even X-ray binaries with lower main sequence donors which simply took longer than the typical few Gyrs (White \& Ghosh 1998) to come into contact. We plan to investigate these possibilities in future work with deeper optical photometry and with spectroscopic follow-ups.

\subsection{Implications for $X$-ray binaries in other stellar populations}

These results clearly indicate that the canonical factor of 100 enhancement in X-ray binaries per unit stellar mass in globular clusters applies only to bright LMXBs. Comparing this galaxy with NGC 6440, which shows the highest number density of quiescent LMXBs (Heinke et al. 2003), we find that the globular cluster density is enhanced by a factor of only about 10, even assuming that we have found all the quiescent LMXBs in Sculptor already. Since other clusters are less rich in LMXBs than NGC 6440, the enhancement factor is even lower on the whole. This is probably because very wide binaries such as those with red giant donors do not survive unperturbed for a Hubble time in dense globular clusters.

Finally, we consider the implications of these results for the Milky Way's bulge's X-ray binary population. The bulge of the Milky Way contains about $5 \times 10^{2}$ times as many stars as the Sculptor dwarf spheroidal galaxy, meaning that it should have about 2,500 
X-ray binaries if the number of binaries scales linearly with mass. In fact, since X-ray binary production is enhanced in metal rich systems (at least for globular clusters - see Kundu, Maccarone \& Zepf 2003 for evidence of this effect and Maccarone, Kundu \& Zepf 2004 for a discussion of a binary evolution model for this effect which should work equally well in field populations as in globular clusters) the Milky Way might contain a few times more than this number of X-ray binaries. Our estimate is comparable to numbers from theoretical predictions (Iben et al. 1997; Belczynski \& Taam 2004).

Finally, we note that with only about 500 optical stars in the Schweitzer et al. (1995) catalog, at least $\approx 1 \%$ of the giant branch/horizontal branch stars in Sculptor are in systems with accreting neutron stars or black holes. Based on this finding, one would expect that a large number of similar systems are likely to be found in surveys that include hundreds of red giant stars. This hypothesis should be testable as the results from the ChaMPlane survey (Grindlay et al. 2003) begin to come in, or in a large area survey of the Galactic Bulge.

\section{Acknowledgements}

We are grateful to Carine Babusiaux, Kyle Cudworth, Peter Jonker, Christian Knigge, Mike Muno, Eric Pfahl, Andrea Schweitzer, Jeno Sokoloski, Kyle Westfall and Rudy Wijnands for useful discussions. We thank Denise Hurley-Keller, Eva Grebel and Mario Mateo for the optical photometric measurement of the brightest X-ray source. AK and SEZ acknowledge support from NASA grants SAO G04-5091X and LTSA NAG-12975. LB acknowledges support from the NSF via grant PHY99-07949.

\section{References}

Belczynski, K. \& Taam, R.E., 2004, ApJ, 616, 1159

Bildsten, L. \& Deloye, C.J., 2004, ApJ, 607L, 119

Brandt, N. \& Podsiadlowski, P., 1995, MNRAS, 274, 461

Garcia, M.R., McClintock, J.E., Narayan, R., Callanan, P., Barret, D., \& Murray, S.S., 2001, ApJ, 553L, 47

Gilfanov, M., 2004, MNRAS, 349, 146

Grindlay, J.E., 1988, IAU Symp. 126, The Harlow-Shapely symposium on Globular Cluster Systems in Galaxies (Dordrecht: Kluwer), 347

Grindlay, J., et al., 2003, AN, 324, 57

Heinke, C.O., Grindlay, J.E., Lugger, P.M., Cohn, H.N., Edmonds, R.D., Lloyd, D.A., \& Cool, A.M., 2003, ApJ, 598, 501

Heinke, C.O., Grindlay, J.E., Edmonds, P.D., Cohn, H.N., Lugger, P.M., Camilo, F., Bogdanov, S., \& Freire, P.C., 2005, ApJ, 625, 796

Hurley-Keller, D., Mateo, M., \& Grebel, E.K., 1999, ApJ, 523L, 25

Iben, I., Tutukov, A.V., \& Fedorova, A.V., 1997, ApJ, 486, 955

Kalogera, V., 1998, ApJ, 493, 368

Kalogera, V. \& Webbink, R.F., 1998, ApJ, 493, 351

Kundu, A., Maccarone, T.J., \& Zepf, S.E., 2003, ApJ, 574, L5

Maccarone, T.J., Kundu, A., \& Zepf, S.E., 2004, ApJ, 606, 430

Mateo, M., 1998, ARA\&A, 36, 435

Paczynski, B., 1976, IAU Symp. 73, Structure and Evolution of Close Binary Systems, ed. P. P. Eggleton, S. Milton, \& J. Whelan (Dordrecht: Reidel), 75

Piro, A.L. \& Bildsten, L., 2002, ApJ, 571L, 103

Podsiadlowski, P., Rappaport, S., \& Pfahl, E.D., 2002, ApJ, 565, 1107

Schweitzer, A.E., Cudworth, K.M., Majewski, S.R., \& Suntzeff, N.B., 1995, AJ, 110, 2747

White, N.E. \& Ghosh, P., 1998, ApJ, 504, L31

White, R.E. III, Sarazin, C.D., \& Kulkarni, S., 2002, ApJ, 571, L23 


\section{Discussion}

DiStefano: For many reasons, I think your conclusions are likely to be correct. But, to play devil's advocate, I'd like to ask about possible degeneracies in the stellar models for the donor stars?

MACCARONE: We have proper motion confirmations that these stars are Sculptor galaxy members from the work of Schweitzer et al. (1995).

SARAZIN: What is the probability of chance overlaps of the X-ray error circles and optical stars in Sculptor?

MACCARONE: For the good matches, the error circles are all less than 0.5 ", so the chance probability of superposition is about 0.02 , if memory serves me correctly. For the fainter sources, because there are more sources and we used larger error circles, we got 4 more matches with a false match probability of about 0.4 , so there's a reasonably large chance that at least one of those is a chance superposition.

SARAZIN: Just a clarification - when you say that GC LMXB production has been overestimated, I assume you are referring only to fainter sources.

MACCARONE: Correct. Another way to look at this is that the duty cycles for the globular cluster sources are strongly enhanced compared to the field sources, and that this, rather than the number density enhancement is the dominant part of the reason why GCs show $\sim 100$ times as many bright X-ray binaries per unit stellar mass as the field stars do.

IVANOVA: It's a comment: You should be a bit more careful making the link between metal-poor dwarf galaxies and metal-rich giant ellipticals, as both the mass transfer rates and formation rates of X-ray binaries with a MS or a RG donors are not the same.

MACCARONE: I agree that these are important considerations, and that my extra polations to larger galaxies should be taken as very rough estimates. 